\title{
An improved fruit transcriptome and the identification of the candidate genes involved in fruit abscission induced by carbohydrate stress in litchi
}

\section{OPEN ACCESS}

Edited by:

Marinus J. M. Smulders, Wageningen University and Research Centre, Netherlands

Reviewed by:

Samira Mafi Moghaddam, North Dakota State University, USA Sushma Naithani,

Oregon State University, USA

*Correspondence:

Jianguo Li,

State Key Laboratory

for Conservation and Utilization of Subtropical Agro-Bioresources, China Litchi Research Center, South

China Agricultural University, 483 Wushan Street, Guangzhou,

Guangdong 510642, China jianli@scau.edu.cn

†These authors have contributed equally to this work.

Specialty section:

This article was submitted to Crop Science and Horticulture, a section of the journal Frontiers in Plant Science

Received: 30 January 2015 Accepted: 28 May 2015

Published: 15 June 2015

Citation:

Li C, Wang Y, Huang $X, L i J$, Wang $H$ and $\mathrm{Li} J$ (2015) An improved fruit transcriptome and the identification of the candidate genes involved in fruit abscission induced by carbohydrate stress in litchi.

Front. Plant Sci. 6:439. doi: 10.3389/fpls.2015.00439

\author{
Caiqin $\mathrm{Li}^{1,2+}$, Yan Wang ${ }^{2,3+}$, Xuming Huang ${ }^{2}$, Jiang $\mathrm{Li}^{3}$, Huicong Wang ${ }^{2}$ and Jianguo Li ${ }^{1,2 *}$ \\ 'State Key Laboratory for Conservation and Utilization of Subtropical Agro-Bioresources, China Litchi Research Center, \\ South China Agricultural University, Guangzhou, China, ${ }^{2}$ Physiological Laboratory for South China Fruits, College of \\ Horticulture, South China Agricultural University, Guangzhou, China, ${ }^{3}$ Beijing Genomics Institute at Shenzhen, Shenzhen, \\ China
}

Massive young fruit abscission usually causes low and unstable yield in litchi (Litchi chinensis Sonn.), an important fruit crop cultivated in tropical and subtropical areas. However, the molecular mechanism of fruit drop has not been fully characterized. This study aimed at identification of molecular components involved in fruitlet abscission in litchi, for which reference genome is not available at present. An improved de novo transcriptome assembly was firstly achieved by using an optimized assembly software, Trinity. Using improved transcriptome assembly as reference, digital transcript abundance (DTA) profiling was performed to screen and identify candidate genes involved in fruit abscission induced by girdling plus defoliation (GPD), a treatment significantly decreased the soluble sugar contents causing carbohydrate stress to fruit. Our results showed that the increasing fruit abscission rate after GPD treatment was associated with higher ethylene production and lower glucose levels in fruit. A total of 2,771 differentially expressed genes were identified as GPD-responsive genes, 857 of which were defined by GO and KEGG enrichment analyses as the candidate genes involved in fruit abscission process. These genes were involved in diverse metabolic processes and pathways, including carbohydrate metabolism, plant hormone synthesis, and signaling, transcription factor activity and cell wall modification that were rapidly induced in the early stages (within 2 days after treatment). qRT-PCR was used to explore the expression pattern of 15 selected candidate genes in the abscission zone, pericarp, and seed, which confirmed the accuracy of our DTA data. More detailed information for different functional categories was also analyzed. This study profiled the gene expression related to fruit abscission induced by carbohydrate stress at whole transcriptome level and thus provided a better understanding of the regulatory mechanism of young fruit abscission in litchi.

Keywords: Litchi chinensis Sonn., transcriptome, carbohydrate stress, fruit abscission, de novo assembly, digital transcript abundance, genes 


\section{Introduction}

Litchi (Litchi chinensis Sonn.) is one of the important economic fruit crops widely grown in south China and Southeast Asian areas. The excessive fruit drop during fruit development is a major problem causing serious economic loss for the growers. Yuan and Huang (1988) reported three to four waves of fruit drop throughout fruit development in different cultivars based on relative abscission rate. Wave I, wave II, and wave III of abscission occurred around 1,3, and 6-7 weeks after full bloom, respectively, but wave IV was specific to cultivars with aborted seeds and occurred 2-3 weeks before harvest. Overall, less than $5 \%$ of the initial female flowers can develop into mature fruit (Stern et al., 1993, 1995; Mitra et al., 2003). Endogenous hormones (Yuan and Huang, 1988; Xiang et al., 1995; Li et al., 2004) and carbohydrates (Yuan and Huang, 1992, 1993; Zhou et al., 1999; Hieke et al., 2002; Chang and Lin, 2007, 2008; Yuan et al., 2009) are proposed to play key roles in the regulation of fruit abscission in litchi. In citrus, it has been suggested that the subsequent fruit development is mostly supported by nutrient supply after hormonal activation of initial fruit growth (Talon et al., 1997). Thus, once mineral and water requirements are satisfied, competition for photoassimilates is thought to be responsible for fruit drop (Moss et al., 1972; Powell and Krezdorn, 1977; Gómez-Cadenas et al., 2000). Defoliation experiments showed that the sucrose status of the fruitlet is a major factor in the regulatory mechanism of fruit abscission in citrus (Mehouachi et al., 1995; GómezCadenas et al., 2000) and apple (Berüter and Droz, 1991). On girdled macadamia branches, the number of fruit retained was dependent on the number of available leaves, which determined the availability of assimilates to the fruit (Trueman and Turnbull, 1994). When applying pedicel girdling at the proximal portion to induce citrus fruitlet drop, reduction of soluble sugars accumulation and induction of ethylene production occurred as well (Iglesias et al., 2006). In litchi, Yuan and Huang (1988) demonstrated that litchi fruit set was strongly dependent on current photosynthesis. Treatment of girdling (a ring of bark and cambium was removed from the branch base) plus defoliation (100\% leaf removal) in litchi reduced endogenous IAA concentration and increased the transcript level of two IAA-responsive genes ( $L c A U X / I A A 1$ and $L c S A U R 1$ ), one cell wall degrading enzyme gene ( $L C P G 1)$ and one ethylene biosynthetic gene ( $L c-A C O 1)$, in contrast to the decreasing accumulation of auxin response factor ( $L c A R F 1)$ mRNA, with the concomitant increase in fruit drop (Kuang et al., 2012; Peng et al., 2013; Wu et al., 2013). However, a deep knowledge of the molecular events involved in fruit abscission induced by carbohydrate stress is still missing.

With the development of microarray and next generation sequencing technology, global transcriptome analyses have been widely used to investigate the molecular regulatory networks on various organs abscission, such as flower (Cho et al., 2008; Meir

Abbreviations: AZ, abscission zone; CFAR, the cumulative fruit abscission rate; $\mathrm{CK}$, control; DEG, differentially expressed gene; DTA, digital transcript abundance; GO, Gene Ontology; GPD, girdling plus defoliation; KEGG, Kyoto encyclopedia of genes and genomes. et al., 2010; Wang et al., 2013), leaves (Agustí et al., 2009), shoot tips (Zhang et al., 2014), young fruit (Botton et al., 2011; Zhu et al., 2011), and mature fruit (Corbacho et al., 2013; Gil-Amado and Gomez-Jimenez, 2013). In litchi, the reference genome is not available at present. We previously assembled the first fruit transcriptome by SOAPdenovo software and discovered 1,039 differentially regulated unigenes responding to shading-induced fruitlet abscission ( $\mathrm{Li}$ et al., 2013). However, other assembly software, such as Trinity and Oases, has been shown to obtain superior overall results compared to SOAPdenovo (Zhao et al., 2011; Ghangal et al., 2013). Here, we firstly used four publicly available assemblers to assess the litchi fruit RNA-Seq data generated previously (Li et al., 2013). And then, we compared GPD-induced fruit drop with the CK via gene expression profiling performed on the three pooled tissues (fruit AZ, pericarp and seed), sampled 2, 4, and 7 days after GPD treatment. A number of pathways and candidate genes associated with fruit abscission were screened and identified. Our results provided more clues for a better understanding of the mechanisms of fruit abscission induced by carbohydrate stress in litchi.

\section{Materials and Methods}

\section{Plant Materials and Treatments}

Three randomly selected 9-years-old litchi trees (Litchi chinensis Sonn. cv. Wuye) grown in an orchard in South China Agricultural University (Guangzhou, China) were chosen. Twenty fruitbearing shoots with similar diameter located in different directions from each tree were tagged. Ten of them were treated with girdling (a ring of bark about $0.5 \mathrm{~cm}$ in width and cambium was removed from the branch base) followed by defoliation (removing all leaves above the girdle) at 35 days after anthesis (GPD treatment), while the remaining untreated shoots were used as CK. Three out of ten treated shoots were used to monitor fruit abscission dynamic and the others were used for sampling. Samples were collected at 0,2, 4, and 7 days after treatment. Fruit were dissected using a sharp razor blade into pericarp and seed, while AZ was excised by cutting around $2 \mathrm{~mm}$ at each side of the abscission fracture plane. After separation, all tissues were quickly frozen in liquid nitrogen and stored at $-80^{\circ} \mathrm{C}$ for future analysis. Each tree was treated as a biological replicate.

\section{Determination of Fruit Abscission and Ethylene Production Rate of Fruit}

Cumulative fruit abscission rate and relative fruit abscission rate were calculated according to our previous method (Kuang et al., 2012). Ethylene production was measured according to the method described by Yan et al. (2011) with some modifications. Two fruit from each treatment on each tree were collected and enclosed in a $25 \mathrm{~mL}$ airtight syringe equipped with a rubber piston for $2 \mathrm{~h}$ at $25^{\circ} \mathrm{C}$. Air within the syringe was forced into an airtight container filled with saturated salt water with a needle inserted to allow replacement. After all the air samples were collected in the experiment, $1 \mathrm{~mL}$ air sample was then withdrawn from the headspace of the container with a syringe and injected into a GC-17A gas chromatograph (Shimadzu, Kyoto, Japan) 
fitted with a flame ionization detector and an activated alumina column $(200 \mathrm{~cm} \times 0.3 \mathrm{~cm})$. The injector temperature was $120^{\circ} \mathrm{C}$; the column temperature was kept at $60^{\circ} \mathrm{C}$ and the detector temperature at $60^{\circ} \mathrm{C}$. Helium was used as carrier gas at a flow rate of $30 \mathrm{~mL} \cdot \mathrm{min}^{-1}$. The ethylene production rate was expressed as microliters of $\mathrm{C}_{2} \mathrm{H}_{4} \mathrm{~kg}^{-1} \cdot \mathrm{h}^{-1}$.

\section{Extraction and Measurement of Sugars}

Sugars were extracted and determined according to the protocol of Hu et al. (2005) with modifications. Pericarp or seed samples (200 mg) were ground in liquid nitrogen with a mortar and then extracted with $5 \mathrm{~mL}$ of $80 \%$ ethanol $(\mathrm{v} / \mathrm{v})$ at $80^{\circ} \mathrm{C}$ for $40 \mathrm{~min}$. The extracts were then centrifuged at $12,000 \mathrm{~g}$ for $10 \mathrm{~min}$ at room temperature. The ethanol extraction step was repeated once. The supernatant resulting from the two extractions was combined and diluted with $80 \%$ ethanol to $10 \mathrm{~mL}$. An aliquot $2 \mathrm{~mL}$ of the supernatant was dried with a rotary evaporator for $8 \mathrm{~h}$ and then dissolved in $2 \mathrm{~mL}$ of distilled water. After dissolution, the supernatant $(1 \mathrm{~mL})$ was passed through a Sep-Pak ${ }^{\circledR} 1 \mathrm{cc}(100 \mathrm{mg})$ C18 cartridge (Waters Corporation, Milford, MA, USA). Sugars were measured by high-performance liquid chromatography (HPLC) using an Agilent 1200 HPLC system (Agilent Technologies, Waldbronn, Germany) with a G1362A refractive index detector cell maintained at $40^{\circ} \mathrm{C}$. A Transgenomic CARB Sep Coregel $87^{\circ} \mathrm{C}$ column (CHO-995860) with a guard column cartridge (Transgenomic CARB Sep Coregel $87^{\circ} \mathrm{C}$ cartridge) was used. The column was maintained at $80^{\circ} \mathrm{C}$ with a thermostatic column compartment. The injection volume was $10 \mu \mathrm{L}$. Samples were eluted with double-distilled water. The flow rate was $0.6 \mathrm{~mL} \cdot \mathrm{min}^{-1}$. The quantification of sugars was performed according to external standard solution calibration (sugar standards were purchased from Sigma Chemical Co.).

\section{Comparison of Litchi Fruit Transcriptome Assembly}

In this study, we compared the performance of four commonly used de novo short read assembly softwares including SOAPdenovo $^{1}$ (version 1.04, Li et al., 2009), SOAPdenovoTrans $^{2}$ (version 1.03, Xie et al., 2014), Velvet-Oases ${ }^{3}$ (Velvet: version 1.2.09, and Oases ${ }^{4}$ : version 0.2.08, Schulz et al., 2012) and Trinity ${ }^{5}$ (version 2013-02-25, Grabherr et al., 2011). These programs have been developed for short reads assembly using a de Bruijn graph algorithm (Zerbino and Birney, 2008). SOAPdenovo-Trans was used to assemble $k$-mer lengths of 2341 with a step length of 4 , Velvet-Oases were used to assemble $k$-mer lengths of 19-43 with a step length of 6, SOAPdenovo and Trinity was used to assemble a single $k$-mer length of 25 . Any redundant fragments generated from four softwares were removed by TGICL (version 2.1) and Phrap (version release 23.0) assembler to get final genes. Following parameters were used to

\footnotetext{
${ }^{1}$ http://soap.genomics.org.cn

${ }^{2}$ http://soap.genomics.org.cn/SOAPdenovo-Trans.html

${ }^{3}$ http://www.ebi.ac.uk/ zerbino/velvet/

${ }^{4}$ http://www.ebi.ac.uk/ zerbino/oases/

${ }^{5} \mathrm{http}: / /$ sourceforge.net/projects/trinityrnaseq
}

ensure a high quality of assembly: a minimum of 95\% identity, a minimum of 35 overlapping bases, a minimum of 35 scores and a maximum of 25 unmatched overhanging bases at sequence ends. Based on sequence similarity, the genes were divided into two classes: clusters (prefixed with 'CL') and singletons (prefixed with 'unigene'). In a cluster, the mutual similarity was more than $70 \%$. Clean reads were mapped back onto respective assembled genes using SOAPaligner (version: 2.21) to check the integrity of assembled results. Total number of genes, N50 and average gene length were also taken into consideration to evaluate the quality of transcriptome assemblies. The best transcriptome assembly was used for following analyses.

Functional annotation of the assembled genes was predicted based on the highest similarity in the following databases: $\mathrm{Nr}^{6}$ (NCBI non-redundant protein sequences), $\mathrm{Nt}^{6}$ (NCBI non-redundant nucleotide sequences), $\mathrm{COG}^{6}$ (Clusters of Orthologous Groups of proteins, COG), Swiss-Prot ${ }^{7}$ (A manually annotated and reviewed protein sequence database), $\mathrm{KO}^{8}$ (KEGG Orthology database) and GO. GO functional annotation was performed by Blast2GO (v2.5.0) software (Conesa et al., 2005). The raw sequence data used in this study has been submitted to National Center for Biotechnology Information (NCBI) Short Read Archive (SRA) with accession number SRX255051 (Li et al., 2013). The sequences assembled by Trinity have been stored in NCBI's Transcriptome Shotgun Assembly (TSA) database which can be accessed using the Gene-IDs listed in Supplementary Table S1.

\section{Digital Transcript Abundance Library Preparation and Illumina Sequencing}

Total RNA from three tissues (AZ, pericarp, and seed) was isolated using Column Plant RNAout 2.0 kit (TIANDZ, Inc., China). The quantity and quality of RNA samples were evaluated using 2100 Bioanalyzer (Agilent Technologies, Santa Clara, CA, USA). Equal total RNA from different tissues sampled at specific time points of the same treatment were pooled to construct six libraries named CK2, CK4, CK7, GPD2, GPD4, and GPD7. For example, CK2 and GPD2 were the libraries from tissues harvested at 2 days in the $\mathrm{CK}$ and the GPD treatment, respectively. After RNA extraction, mRNA purification by Oligo (dT), fragmentation, cDNA synthesis by random hexamer primers, size selection and PCR amplification were sequentially performed by BGI-Shenzhen as described previously (Li et al., 2013). DTA datasets were deposited in the NCBI's SRA database with the accession numbers as follows: SRX847812 (CK2), SRX847822 (CK4), SRX847823 (CK7), SRX847824 (GPD2), SRX847825 (GPD4), and SRX847826 (GPD7).

\section{Data Analysis for Digital Transcript Abundance Profiles}

High-quality reads filtered through the standard Illumina pipeline to remove the low-quality reads and those containing adaptor/primer contaminations were used for further

\footnotetext{
${ }^{6}$ http://www.ncbi.nlm.nih.gov

${ }^{7}$ http://www.expasy.ch/sprot

${ }^{8}$ http://www.genome.jp/kegg
} 
downstream processing. All clean reads were mapped back to the updated transcriptome reference database using SOAPaligner (version 2.21) allowing up to two nucleotide mismatches with the parameters of " $-\mathrm{m} 0-\mathrm{x} 1000-\mathrm{s} 28-132-\mathrm{v} 2-\mathrm{r} 2$," which are specified on http://soap.genomics.org.cn/soapaligner.html. For gene expression analysis, the number of unambiguous clean reads for each gene was calculated and normalized to RPKM (Reads Per Kilobase per Million reads; Mortazavi et al., 2008). Three paired-libraries including CK2 vs. GPD2, CK4 vs. GPD4, and CK7 vs. GPD7 were used to analyze the differential gene expression, according to the method described in Audic and Claverie (1997). Two filter criteria were used to identify DEGs: a fold change in transcript levels $\geq 4$ and a FDR (False Discovery Rate) value $\leq 0.001$. For functional and pathway-enrichment analyses, all DEGs were mapped to terms in GO and KEGG databases. Heatmaps showing expression profiles ( $\log _{2}$ fold change) were generated using the MultiExperiment Viewer ( $\mathrm{MeV}, \mathrm{v} 4.9)$. Hierarchical clustering was performed by Euclidean distance matrix with a complete linkage rule using MeV. DEGs significantly enriched in GO term analysis (FDR $\leq 0.05$ ) or enriched in KEGG pathway ( $Q$ value $\leq 0.05$ ) were screened to be the candidate genes involved in the fruit abscission process.

\section{Quantitative Real-Time PCR Analysis}

To validate the accuracy of our DTA profiling results, 15 randomly selected candidate genes were evaluated by quantitative real-time PCR (qRT-PCR) after GPD-treatment in AZ, pericarp, and seed of litchi. The list of gene-specific primers was shown in Supplementary Table S2. RNA reverse transcription, qRT-PCR reaction, data normalization, and calculation were performed as described previously (Li et al., 2013). Values of each time point were means of three biological replicates.

\section{Results}

\section{Transcriptome Assembly Optimization}

Here, four publicly available assemblers were used to assess the litchi fruit RNA-Seq data generated previously (Li et al., 2013). For SOAPdenovo-Trans, increased $k$-mer value (ranging from 23 to 41 ) led to the quality deterioration in the generated assembly with the reduction of average and N50 gene lengths, so the 23-mer in SOAPdenovo-Trans was selected for final assembly. In contrast, assembly generated by Velvet-Oases at $k=31$ was found to obtain better quality when different $k$-mer lengths ranging from 19 to 43 (Supplementary Figure S1) were tested. Compared to other three assembly software, Trinity generated the best assembly with the largest average (776 bp) and N50 (1,198 bp) gene lengths and utilized $88.70 \%$ of the total reads, SOAPdenovo-Trans $(k=23)$ came in the second, and SOAPdenovo used in our previous study was the worst in the category due to the lowest utilized reads (Table 1). Moreover, the assembly programs varied widely in the lengths distributions of the genes. Trinity produced superior results in much larger numbers of long-genes $(>1 \mathrm{~kb})$ and fewer shorttranscripts (200-500 bp; Supplementary Figure S2A). Thus, the
TABLE 1 | Comparison of different assembled softwares.

\begin{tabular}{lllll}
\hline & SOAPdenovo & $\begin{array}{l}\text { SoAPdenovo- } \\
\text { Trans } \\
(\boldsymbol{k}=\mathbf{2 3})\end{array}$ & $\begin{array}{l}\text { Velvet- } \\
\text { oases } \\
(\boldsymbol{k}=\mathbf{3 1})\end{array}$ & Trinity \\
\hline Number of genes & 57,050 & 32,455 & 59,461 & 45,370 \\
Maximum length (bp) & 10,687 & 8,220 & 4,979 & 9,098 \\
Average length (bp) & 601 & 679 & 442 & 776 \\
GC (\%) & 40.88 & 43.09 & 42.57 & 42.42 \\
N (\%) & 1.37 & 0.07 & 0.50 & 0 \\
N50 length (bp) & 811 & 997 & 491 & 1,198 \\
Reads utilization (\%) & 39.32 & 73.26 & 60.69 & 88.70 \\
\hline
\end{tabular}

transcriptome assembled by Trinity was used for functional annotation. This improved assembly had 45,370 non-redundant genes without ' $\mathrm{N}$ ', comprising a total length of $35,197,676 \mathrm{bp}$, with 22,706 genes (50.05\%) longer than 500 bp, 11,932 genes $(26.30 \%)$ longer than $1000 \mathrm{bp}$, and 2,918 (6.43\%) genes longer than $2000 \mathrm{bp}$ (Supplementary Figure S2A). To evaluate the accuracy of the assembled sequence, all the usable reads were re-aligned onto the genes (Supplementary Figure S2B), showing that there were 42,661 genes $(94.03 \%)$ with very high reads coverage $(90 \sim$ $100 \%)$.

\section{Changes in Fruit Abscission Rate, Ethylene Production, and Sugars Contents in Response to GPD Treatment}

Cumulative fruit abscission rate and ethylene production in fruit were compared between the GPD treatment and the CK (Figure 1). The CFARs showed similar trends (Figure 1A), which gradually increased during the first 2 days and had no visible difference. Four days after treatment, the CFAR in GPD-treated fruit was significantly higher than that in the CK. Consequently, $88.0 \%$ of the fruit had dropped by 7 days after the GPD treatment, in contrast to the $28.0 \%$ loss in the CK, indicating that GPD treatment significantly accelerated fruit drop. In addition to the induction of fruit drop, a clear impact on ethylene production was also observed in GPD treated fruit. Within the 7 days of observation, ethylene production of CK fruit remained at a low level below $2 \mu \mathrm{l} \cdot \mathrm{kg}^{-1} \cdot \mathrm{h}^{-1}$. In contrast, ethylene production of GPD treated fruit increased rapidly and

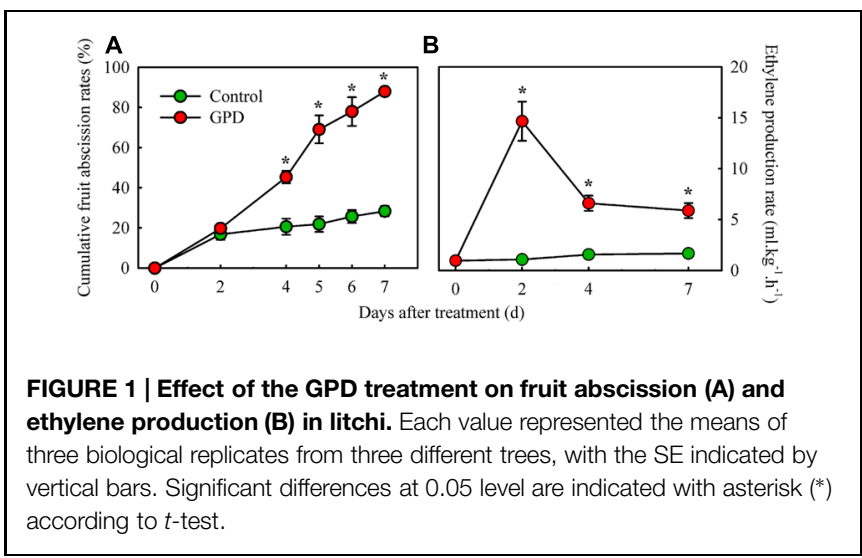


became 15 -fold higher at days 2, then decreased slightly but remained higher level than the CK (sixfolds higher than the CK) until 7 days after treatment (Figure 1B). The increase in ethylene production suggested that the GPD treatment probably accelerated fruit drop through the induction of fruit ethylene production.

Girdling plus defoliation treatment also led to obvious changes in soluble sugar contents in fruit. The contents of sucrose, glucose, and fructose were determined in both the pericarp and seed tissues. The sucrose content in the GPD treated fruit decreased more quickly, and therefore was significantly lower than those in the $\mathrm{CK}$ from 2 to 7 days after treatment in both tissues (Figures 2A,B). Although the glucose and fructose concentrations showed a similar change pattern, both of them in CK were significantly higher than those in GPD treated fruit from 2 to 7 days in pericarp (Figures 2C,E), and at 7 days in seed (Figures 2D,F). These results indicated that blocking carbon nutrient supply to fruit by the GPD treatment caused decrease in soluble sugar contents in fruit, generating carbohydrate stress which might aggravate the fruit abscission.

\section{Digital Transcript Abundance Profile Analysis and Clustering of Differentially Expressed Genes}

To explore the transcriptional changes of litchi fruit in response to carbohydrate stress induced by the GPD treatment, six DTA tag profile libraries of the CK and GPD treated tissue samples (CK2, CK4, CK7, GPD2, GPD4, and GPD7) were constructed

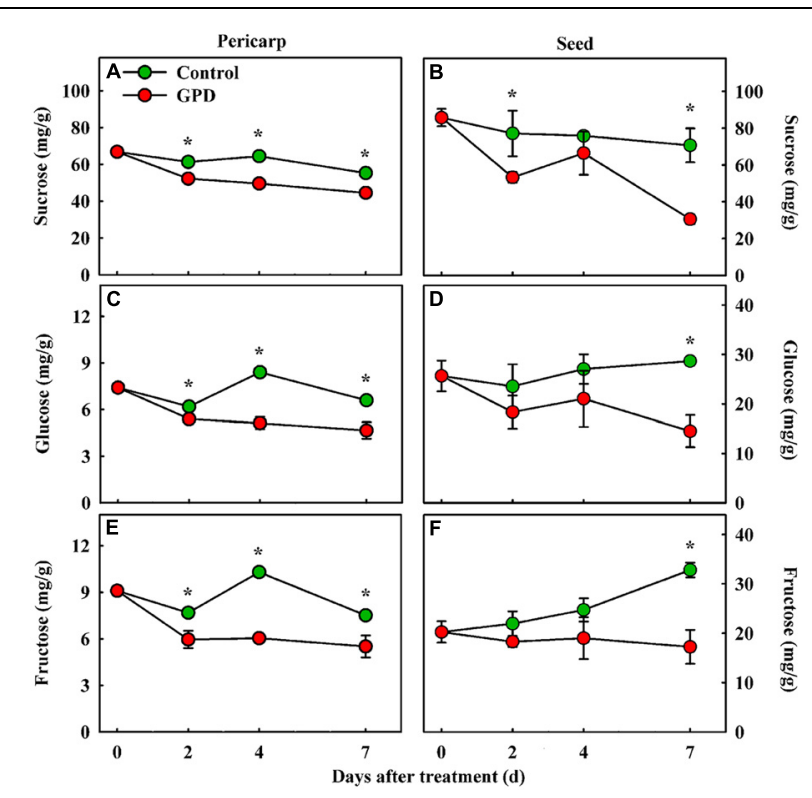

FIGURE 2 | Change in soluble sugar contents (sucrose, glucose, and fructose) in the fruit pericarp (A,C,E) and seed (B,D,F) of litchi after the GPD treatment. Each value represented the means of three biological replicates from three different trees, with the SE indicated by vertical bars. Significant differences at 0.05 level are indicated with asterisk $(*)$ according to t-test.
TABLE 2 | Statistics of DTA libraries.

\begin{tabular}{llll}
\hline & Total reads & Total mapped reads* & Unique match reads \\
\hline CK2 & $6,053,801$ & $5,092,795(84.13 \%)$ & $4,258,546(70.34 \%)$ \\
CK4 & $5,064,253$ & $4,120,052(81.36 \%)$ & $3,450,681(68.14 \%)$ \\
CK7 & $5,225,695$ & $4,253,551(81.40 \%)$ & $3,599,995(68.89 \%)$ \\
GPD2 & $5,897,341$ & $5,032,315(85.33 \%)$ & $4,373,685(74.16 \%)$ \\
GPD4 & $5,898,762$ & $5,255,458(89.09 \%)$ & $4,655,256(78.92 \%)$ \\
GPD7 & $5,898,257$ & $5,047,765(85.58 \%)$ & $4,483,144(76.01 \%)$ \\
\hline
\end{tabular}

*Number and percentage of reads mapped onto litchi fruit transcriptome assembled by Trinity.

and sequenced (Table 2). After quality filtering, a total of 34 million reads were generated from the above six libraries (56 million reads for each library). The tag sequences of the six libraries were mapped to the litchi fruit transcriptome assembled by Trinity, and $81-89 \%$ of all clean reads were matched (Table 2 ), suggesting that we obtained a good quality of sequencing DTA libraries.

After comparing the three paired-libraries (CK2 vs. GPD2, CK4 vs. GPD4, and CK7 vs. GPD7), a total of 2,771 DEGs were identified (Supplementary Table S3). Among which, 1,110, 1,368, and 866 DEGs were down-regulated and 180, 92, and 80 were up-regulated in GPD2/CK2, GPD4/CK4, and GPD7/CK7, respectively (Supplementary Figure S3A). GPD2/CK2 and GPD4/CK4 had more DEGs than GPD7/CK7, suggesting that more genes were differentially regulated at the early and middle stages ( 2 and 4 days) of carbohydrate stress treatment. Venn diagram analysis and a hierarchical clustering showed significant differences in the gene expression profiles between GPD2/CK2 and GPD4/CK4 or between GPD4/CK4 and GPD7/CK7, in contrast to the relatively high similarity between GPD2/CK2 and GPD7/CK7 (Supplementary Figures S3B,C). Noteworthy, a total of 162 DEGs (27 and 135 genes were up- and down-regulated, respectively) were shared in the three comparisons and may represent typical GPD responsive genes.

Based on similar kinetic patterns of expression, all 2,771 DEGs were classified into 14 types of clusters. They could be further divided into four groups based on their temporal pattern of expression (Figure 3). Group I included 1,128 early-responsive genes whose expression were up- or down-regulated early at 2 days after treatment; Group II had 1,129 middle-responsive genes whose expression were not induced or suppressed until 4 days after treatment; Group III contained 353 late-responsive genes that were not regulated until 7 days after treatment; Group IV consisted of 162 constant-responsive genes that up- or downregulated early and whose expression was maintained constant during the treatment. The genes in Group I and Group IV were considered to function in the early stage of fruit abscission, while Group II and Group III were regulators of the late stage.

\section{Analysis of the Candidate Genes Involved in Fruit Abscission}

According to the results of GO and KEGG enrichment analysis, 907 and 1,124 DEGs were identified in GO (FDR $\leq 0.05$ ) and KEGG pathway $(Q$ value $\leq 0.05)$ with significant 


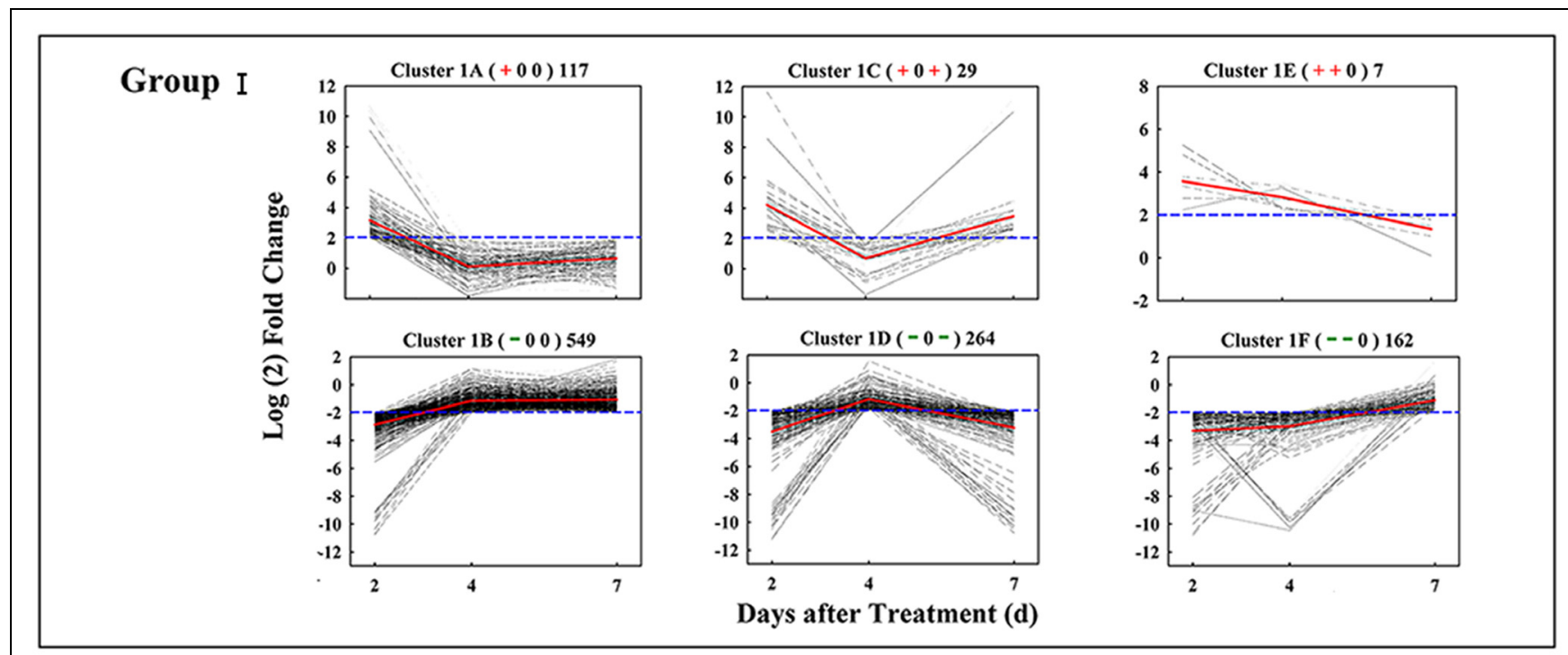

\section{Group II}

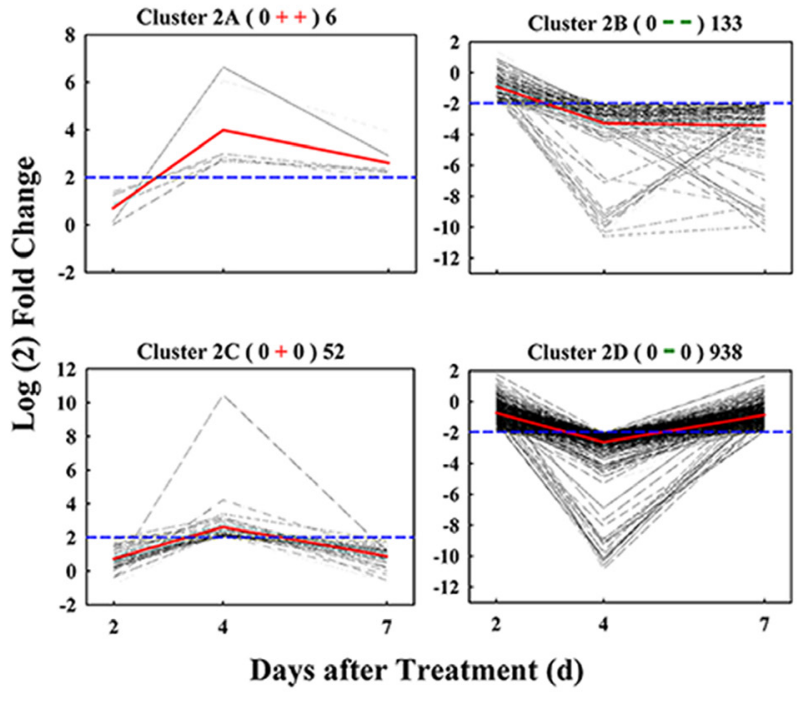

FIGURE 3|Gene expression pattern obtained by kinetics-based clustering. Group I, cluster of DEGs with early and transient changes after GPD-treatment; Group I, clusters of genes modified in their expression until 4 days after GPD-treatment; Group III, cluster of genes with expression kinetics exhibiting late changes after GPD-treatment; Group IV, cluster of DEGs with persistent changes during the whole

\section{Group III}

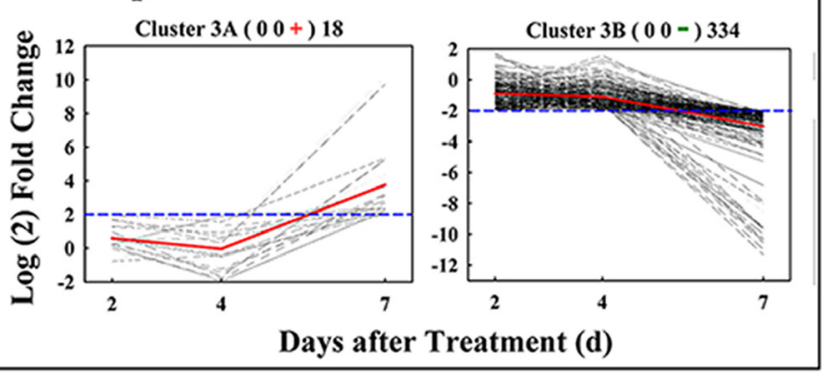

Group IV

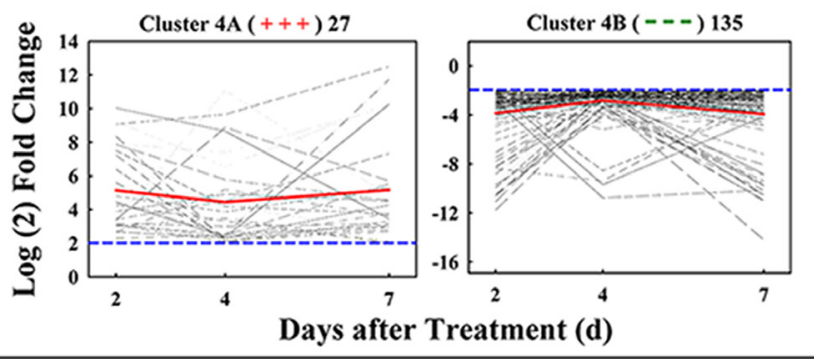

abscission process. The + and - signs in bracket represent up- and down-regulated of genes, respectively, while 0 represents no change. The numbers on the right of bracket indicate the total numbers of DEGs in each cluster. All of these changes were based on a fourfold change criterion (log2 ratio) indicated in the blue dotted lines. Average values of gene-expression level in clusters were showed in the red solid lines. enrichment, respectively, (Supplementary Table S4). After eliminating duplicated genes, 857 of the 2,771 DEGs were identified as the candidate genes involved in fruit abscission process induced by the GPD treatment. The up- and downregulated genes accounted for 13.6 and $86.7 \%$, respectively. According to function annotation, these candidate genes significantly affected by the GPD treatment were divided into 17 functional categories (Table 3; Supplementary Table S5).

\section{Genes Involved in Carbohydrate Metabolism}

Seventy-seven carbohydrate metabolism genes were found to be associated with glycolysis, starch, and sucrose degradation, transferase, gluconeogenesis. There were 57 genes expression down-regulated, and among them, 18, 23, 13, and 3 genes belonged to Group I, Group II, Group III, and Group IV, respectively. Of these, many are involved in glycolysis and starch synthesis such as pyruvate dehydrogenase, ATP-citrate synthase, alcohol dehydrogenase, and sucrose phosphate synthase (SPS), as 
TABLE 3 | Functional categorization of GPD-responsive DEGs.

\begin{tabular}{|c|c|c|c|c|c|c|c|}
\hline \multirow[t]{2}{*}{ Functional categories } & \multirow[t]{2}{*}{ Genes number } & \multirow[t]{2}{*}{ Regulation } & \multicolumn{4}{|c|}{ Group } & \multirow[t]{2}{*}{ Description } \\
\hline & & & $\mathbf{I}$ & II & III & IV & \\
\hline \multirow[t]{2}{*}{ Carbohydrate metabolism } & 77 & Up & 13 & 2 & 0 & 5 & Sugar degradation, glycolysis, gluconeogenesis \\
\hline & & Down & 18 & 23 & 13 & 3 & Sucrose synthesis, glycolysis, transferase, sugar degradation \\
\hline \multirow[t]{2}{*}{ Chloroplast/photosynthesis } & 18 & Up & 0 & 8 & 0 & 0 & PSI/II activity, cytochrome b complex \\
\hline & & Down & 2 & 6 & 2 & 0 & Carbon fixation \\
\hline \multirow[t]{2}{*}{ Energy/mitochondria } & 16 & Up & 0 & 10 & 0 & 0 & Electron transport chain \\
\hline & & Down & 3 & 2 & 1 & 0 & Alternative oxidase \\
\hline \multirow[t]{2}{*}{ Hormone response } & 71 & Up & 5 & 0 & 1 & 1 & Ethylene, gibberellin \\
\hline & & Down & 36 & 10 & 13 & 5 & Abscisic acid, auxin, brassinosteroids, cytokinin, Jasmonic acid \\
\hline \multirow[t]{2}{*}{ Cell wall modification } & 144 & Up & 9 & 0 & 1 & 3 & Degradation, \\
\hline & & Down & 66 & 37 & 18 & 10 & Biosynthesis, degradation, loosening \\
\hline \multirow[t]{2}{*}{ Transcription factor (TF) } & 30 & Up & 2 & 0 & 0 & 0 & HEC, LBD \\
\hline & & Down & 12 & 10 & 5 & 1 & AP2/ERF, bHLH, LBD, GRAS \\
\hline \multirow[t]{2}{*}{ Signal transduction } & 78 & Up & 1 & 0 & 0 & 0 & LRR \\
\hline & & Down & 29 & 31 & 7 & 10 & LRR, CLV, PERK, PI4KB \\
\hline \multirow[t]{2}{*}{ Cytoskeleton/intracellular transport } & 40 & Up & 0 & 1 & 0 & 0 & Proton \\
\hline & & Down & 9 & 24 & 3 & 3 & ABC transporter, microtubule, proton \\
\hline \multirow[t]{2}{*}{ Cell cycle } & 6 & Up & 0 & 0 & 0 & 0 & - \\
\hline & & Down & 3 & 2 & 1 & 0 & Cell division, cyclin \\
\hline \multirow[t]{2}{*}{ Apoptosis/proteolysis } & 15 & Up & 0 & 0 & 0 & 1 & - \\
\hline & & Down & 5 & 6 & 3 & 0 & Protein ubiquitination, protein degradation \\
\hline \multirow[t]{2}{*}{ Oxidation/reduction } & 52 & Up & 7 & 2 & 0 & 1 & POD \\
\hline & & Down & 26 & 10 & 4 & 2 & Rboh, POD, LAC, AO, Rboh \\
\hline \multirow[t]{2}{*}{ DNA/RNA/protein } & 39 & Up & 1 & 0 & 0 & 0 & \\
\hline & & Down & 7 & 25 & 4 & 2 & \\
\hline \multirow[t]{2}{*}{ Stress/pathogenesis } & 49 & Up & 9 & 2 & 2 & 2 & Chitinase, PR gene \\
\hline & & Down & 17 & 14 & 3 & 0 & R gene, lectin \\
\hline \multirow[t]{2}{*}{ Amino acid metabolism } & 32 & Up & 7 & 0 & 0 & 0 & Asparagine synthetase, cysteine synthase \\
\hline & & Down & 13 & 9 & 3 & 0 & Amino acid metabolic process \\
\hline \multirow[t]{2}{*}{ Lipid metabolism } & 48 & Up & 2 & 0 & 0 & 0 & - \\
\hline & & Down & 23 & 21 & 2 & 0 & Lipid/fatty acid synthesis and catabolism \\
\hline \multirow[t]{2}{*}{ Secondary metabolism } & 112 & Up & 11 & 0 & 3 & 1 & Alkaloid, flavonoid \\
\hline & & Down & 53 & 24 & 15 & 5 & Flavonoid, phenolics, phenylpropanoid, terpenoid, vitamin \\
\hline \multirow[t]{2}{*}{ Others } & 30 & Up & 2 & 0 & 1 & 2 & - \\
\hline & & Down & 11 & 7 & 6 & 1 & Cytochrome P450 \\
\hline
\end{tabular}

A total of 857 GPD-responsive DEGs were classified into 17 functional categories.

well as various classes of glycosidase and transferase. In addition, three genes encoding trehalase-6-phosphate phosphatase and trehalose phosphate synthase are also included. In contrast, only 20 carbohydrate metabolism genes were up-regulated. These results indicated that carbohydrate metabolism and sugar signaling pathway were largely inhibited during the entire GPD treatment.

\section{Photosynthesis and Energy/Mitochondria Related Genes}

We found 18 photosynthesis and 16 energy/mitochondria related genes, 10 and 6 of them were down-regulated, respectively, while the remaining genes were up-regulated. Over $70 \%$ genes belonged to Group II, indicating that most genes of these two groups were largely affected at 4 days after the GPD treatment. The expression of genes related to carbon fixation and alternative oxidase was repressed, while the expression of genes associated with PSI and PSII activities, cytochrome b complex and electron transport chain of mitochondria was induced.

\section{Plant Hormone Pathway}

Seventy one genes were found to be related to plant hormone synthesis and signaling pathways, including those related to auxin (IAA, 17 genes), abscisic acid (ABA, 14 genes), ethylene (13 genes), cytokinin (10 genes), brassinosteroid (BR, 8 genes), gibberellin (GA, 5 genes), and jasmonic acid (JA, 4 genes). Among them, 41, 10, 14, and 6 genes belonged to Group I, Group II, Group III, and Group IV, respectively. Five 1-aminocyclopropane-1-carboxylate oxidase $(A C O)$ genes and another two genes encoding carboxylesterase were induced during the early fruit abscission process after the GPD 
treatment, and the other six genes were down-regulated. Taking all 17 IAA-related genes as an example, six out of eight genes encoding auxin induced proteins ( $A u x / I A A)$ and two genes encoding indole-3-acetic acid-amido synthetase $(G H 3)$ were immediately down-regulated at 2 days after the GPD treatment. Five genes encoding ARFs were also repressed at 2 days or 4 days after the GPD treatment. Two genes related to polar auxin transport (auxin influx carrier protein) were affected at 7 days after the GPD treatment. These results indicated that most plant hormone related genes were down-regulated at the early stage of the GPD treatment.

\section{Responses of Cell Wall Modification}

One-hundred and forty four candidate genes, which formed the largest functional category affected by the GPD treatment in our study, were found to be associated with cell wall biosynthesis, loosening, degradation, and modification. Among them, not only all transcripts related to cell wall biosynthesis such as cellulose synthase, but also numerous cell wall degradation and loosening related genes like extensin and pectinesterase were found to be down-regulated. There were only 12 genes associated with cell wall degradation including $\beta$-1,3-glucanase, $\beta$-D-xylosidase, endoglucanase, xyloglucan endotransglucosylase/hydrolase, pectinesterase, polygalacturonase, and $\beta$-D-xylosidase were induced.

\section{Expression of Transcription Factor Genes}

A total of 30 candidate genes were identified as TFs, including AP2/ERF, bHLH, GRAS, LOB domain protein, et cetera. Of them, 14, 10, 5, and 1 genes belonged to Group I, Group II, Group III, and Group IV, respectively, indicating most genes were largely affected at 2 days after the GPD treatment. Apart from $L O B$ domain-containing protein and TF HEC1 in Group I, the remaining 28 genes were down-regulated, suggesting that the vast majority of TFs displayed a repressed expression.

\section{Genes Involved in Signal Transduction and Membrane/Cytoskeleton/Intracellular Transport}

Seventy-eight GPD-responsive DEGs were found to be associated with signal transduction. Except one gene encoding serine/threonine-protein kinase, the expression of all other 77 genes was repressed. Among them, 29, 31, 7, and 10 genes belonged to Group I, Group II, Group III, and Group $\mathrm{IV}$, respectively, indicating most genes were at low level of expression at 2 and 4 days after the GPD treatment. Of these, many were encoding for LRR receptor-like serine/threonineprotein kinase, receptor protein kinase CLAVATA1 and proline-rich receptor-like protein kinase. On the other hand, 40 genes were found to be involved in cytoskeleton or intracellular transport. Among them, all genes were repressed except one gene encoding for $\mathrm{H}^{+}$-transporting two-sector ATPase. These repressed genes included 22 ATP-bind cassette transporters (ABC transporters) and a class of genes related to cytoskeleton function, including those encoding CLIP-associated proteins, formin-like proteins and microtubule-associated proteins.

\section{Impacts on Cell Cycle and Proteolysis}

Our results showed that only six genes were found to be associated with cell cycle, including two cyclin D genes (Group I), one cyclin-dependent kinase inhibitor gene (Group III), and three cell division related genes (Group I and II). They were largely repressed by the GPD treatment. Moreover, 15 genes involved in proteolysis were also found to be down-regulated. These genes encoded F-box proteins, U-box proteins and other members of the ubiquitin ligase complex in potential ubiquitylation pathway, like E3 ubiquitin-protein ligase and the RING-H2 finger protein. A few genes related to protein hydrolysis, such as subtilisin-like protease and serine carboxypeptidase, were repressed as well.

\section{Responses of Stress/Pathogenesis and Oxidation/Reduction}

There were 49 genes involved in stress/pathogenesis response pathway. Among them, 26, 16, 5, and 2 genes belonged to Group I, Group II, Group III, and Group IV, respectively, indicating most genes showed great difference in expression at the early stage. Of them, 11 genes exhibited abrupt upregulated expression at 2 days, including those encoding chitinase, pathogenesis-related proteins, and wound-induced proteins. Whereas, most of the other remaining genes were down-regulation at early or late fruit abscission.

Fifty-two genes were found to be involved in oxidation/reduction processes. Among them, 33, 12, 4, and 3 genes belonged to Group I, Group II, Group III, and Group IV, respectively. Of 10 up-regulated genes, seven genes showed increased transcript abundance at 2 days after the GPD treatment, including those encoding peroxidase, inositol oxygenase, and thioredoxin. Forty-two genes with inhibited expression included genes encoded for laccases (13 genes), peroxidases (10 genes), respiratory burst oxidase homolog proteins (three genes), glutaredoxins (four genes), and L-ascorbate oxidases (three genes).

\section{Validation of the Candidate Genes by qRT-PCR}

In order to verify the expression pattern of those genes involved in fruit abscission, seven up-regulated and eight down-regulated genes were selected for further qRT-PCR analysis. These genes belonged to divergent functional categories or pathways. Six genes (ACO1, ACO2, AUX, Aux/IAA, NCED, and CCD) were implicated in plant hormones (ethylene, auxin, and abscisic acid) regulation pathway, four genes $(P G 1, P G 2, E G$, and $X Y L)$ were involved in cell wall modification, the remaining five genes (SPS, $A P 2 / E R F, U-b o x$, and CHI1, CHI2) were assigned to carbohydrate metabolism, TFs, potential ubiquitylation and stress response. Figure 4 showed that the expressions of $A C O 1$ and $A C O 2$ were increased and $A U X$ and $C C D$ transcript abundance were decreased in three tissues (AZ, pericarp and seed) at 2 and 7 days after the GPD treatment; the expressions of NCED in AZ and $A U X / I A A$ in pericarp were also depressed. The transcript mRNAr level of $P G 1, E G, X Y L, C H I 1$, and $C H I 2$ were increased and $P G 2, S P S, A P 2 / E R F$, and $U-b o x$ were inhibited in all the tissues (Figures 5 and 6). Although the exact fold changes of the selected genes at several data points varied between DTA profile and 

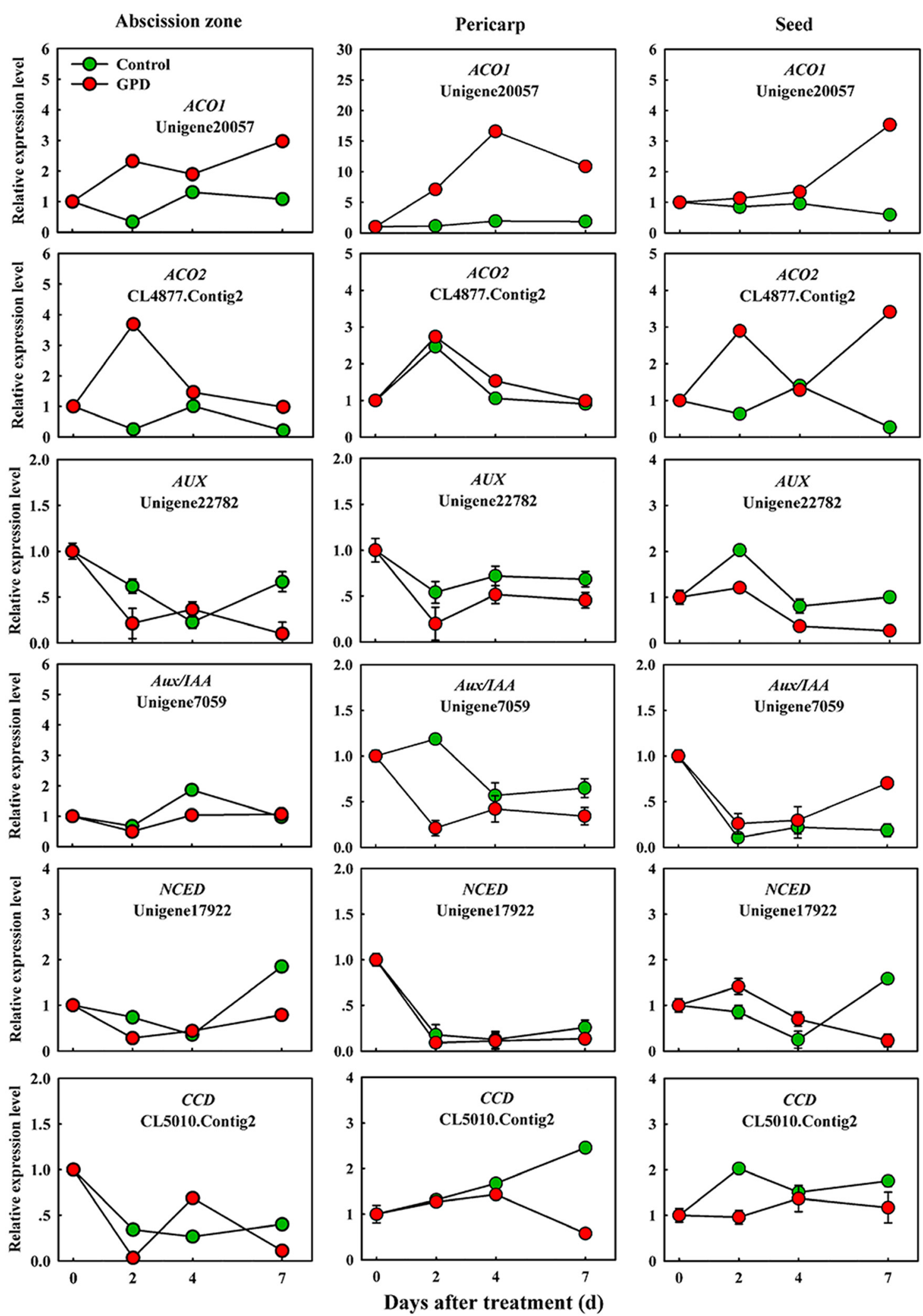

FIGURE 4 | Real-time quantitative PCR analysis of the expression of GPD-responsive DEGs related to hormone signaling and metabolism. The results are means of three biological replicates $( \pm \mathrm{SE})$. The value of transcript levels in CK was arbitrarily set to 1. 

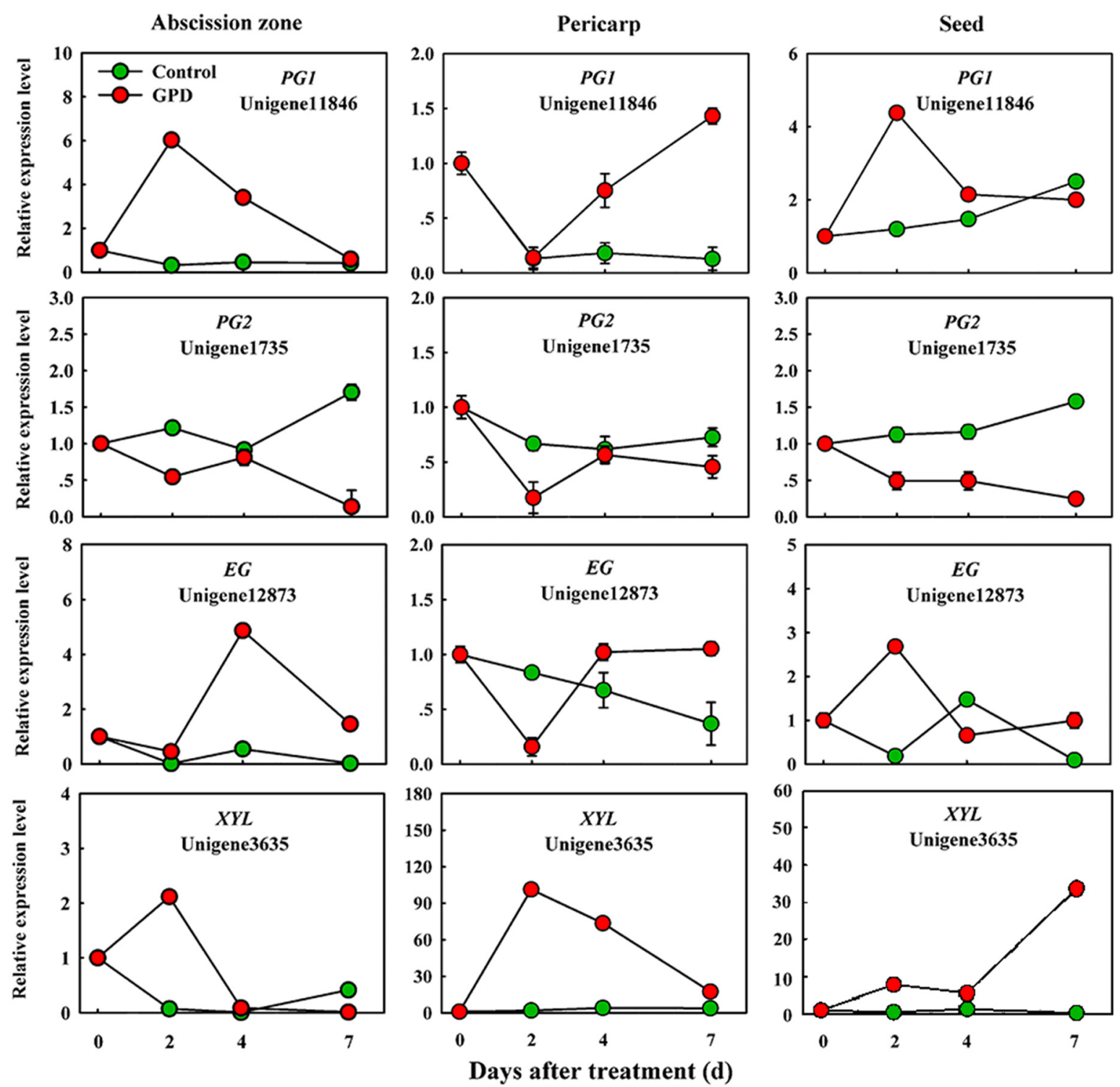

FIGURE 5 | Real-time quantitative PCR analysis of the expression of GPD-responsive DEGs related to cell wall degradation. The results are means of three biological replicates ( \pm SE). The value of transcript levels in CK was arbitrarily set to 1.

qRT-PCR analysis, their expression trends found from the two different approaches were similar. These results re-confirmed the accuracy of our DTA profile data and indicated that AZ was the more important tissue than pericarp and seed during the fruit abscission caused by the GPD treatment in litchi.

\section{Discussion}

Fruit is a heterotrophic organ dependent mainly on the supply of photosynthetic products from leaves. A strong connection between the carbohydrate availability for fruit and their probability of abscission has been described in citrus (Ruiz et al., 2001; Iglesias et al., 2006), apple (Zhu et al., 2011), and also litchi (Yuan and Huang, 1988). In this study, almost $90 \%$ of the fruits on branches dropped after treated by GPD for 7 days, about three times higher than the CK. In the meantime, GPD also significantly decreased the content of sugars and increased ethylene production in fruit. GPD completely blocks the supply of carbohydrate to fruit, which has been proved to be reliable and repeatable experimental model for the research of fruit abscission under carbohydrate stress in litchi (Kuang et al., 2012; Peng et al., 2013). Our previous studies used this experimental model to verify few cloned genes related to auxin signaling (Kuang et al., 2012), cell wall degradation (Peng et al., 2013) and ethylene biosynthesis (Wu et al., 2013) involved in litchi fruitlet drop. Our present work aimed at identification of molecular components involved in fruit abscission in litchi, an important fruit crop, for 


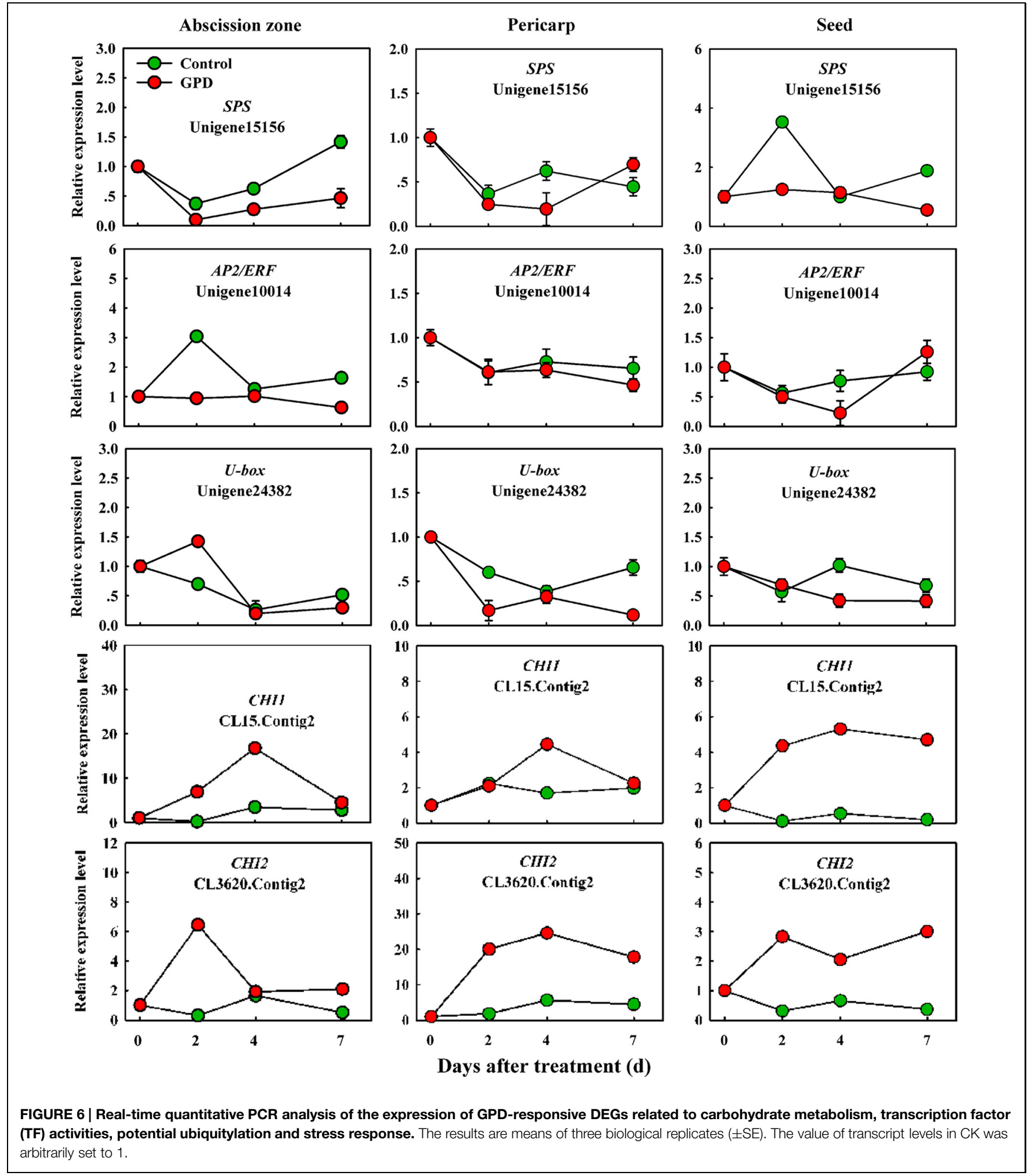

which reference genome is not available now. Thus, this study provided the first report of gene expression profile related to fruit abscission in response to carbohydrate stress on the whole transcriptome level in litchi.
RNA-Seq has been successfully used to sequence the transcriptome of many non-model organisms. De novo transcriptome assembly from short reads is improving with the development of advanced bioinformatics softwares (Schulz 
et al., 2012). In this study, four publicly available assemblers were used to evaluate the litchi fruit RNA-Seq data generated previously ( $\mathrm{Li}$ et al., 2013). Trinity resulted in a better de novo transcriptome assembly compared to other software tested, while previously used SOAPdenovo performed the worst due to its lowest utilized reads. Similar results were reported by Garg et al. (2011) and Zhao et al. (2011). It is not surprising, but having a better assembly for litchi transcriptome is worthwhile and highly desirable. A total of 2,771 significantly DEGs were screened as GPD-responsive genes and 857 of them were identified as the candidate genes involved in fruit abscission process. In general, abscission is considered to develop through four major steps: the $\mathrm{AZ}$ differentiation, the competence to abscission signals, the activation of abscission, and the differentiation of a protective layer (Estornell et al., 2013). In litchi, differentiation of fruit AZ may have occurred very early during flower differentiation (unpublished data). And the second abscission step may occur 0-2 days after the GPD treatment, which might be considered as the early phase of GPD-induced fruit abscission. In this phase, CFAR in the GPD treatment was slowly increased and almost same as the CK, but ethylene release was rapidly risen and significantly higher than the CK. Meanwhile, the expression of 403 and 58 genes belonging to Group I and Group IV, respectively, were changed, which probably leads to acquisition of abscission sensitivity and competence. Therefore, all these genes related to carbohydrate metabolism, hormone response, TFs, and kinase activities, could be directly response to GPD and play an important role on fruit abscission. From 2 to 7 days after treatment, the CFAR in the GPD treatment was largely aggravated at an increasing rate and significantly higher than that in the $\mathrm{CK}$, indicating fruit abscission was being activated and executed. It might be regarded as the late phase of GPD-induced fruit abscission, involving changes in expression of genes in Group II and Group III. A key step in this stage is cell separation, which is mostly induced by cell wall degrading enzymes such as polygalacturonase.

With carbohydrate supply being cut off, it is not surprising that a sharp decline in the soluble sugars level in pericarp and seed occurred after GPD treatment. A reduction of sugar concentration was already reported as a reaction to sugar starvation in citrus (Gómez-Cadenas et al., 2000; Iglesias et al., 2006). In the early phase of the abscission process, we observed a high expression level of genes involved in starch and sucrose degradation, glycolysis and gluconeogenesis, and a decrease in the expression of genes for sucrose synthesis, such as SPS. While in the late phase of the abscission process, a high expression level of genes related to energy metabolism and PSI/II activity and a low expression level of genes associated with carbon fixation and carbohydrate metabolism occurred. These results implicated the reduction in sink storage of GPD-treated fruit and the energy consumption for maintenance. The high expression of carbohydrate metabolism genes was also found in apple (Zhu et al., 2011) and litchi (Li et al., 2013) after shading treatment, while the photosynthesis related genes were inhibited. However, sorbitol metabolism seemed to be more important in apple fruitlet drop (Zhu et al., 2011), which was not found in litchi.
Although the relationships among the hormonal signals, carbohydrate shortage and abscission are still not clearly elucidated, it is widely believed that the endogenous balance of ethylene and auxin in the AZ affects organ abscission. In this study, ethylene production increased and peaked in GPDtreated fruit, coinciding with the up-regulation of genes encoding ethylene biosynthesis ( $A C O 1$ and $A C O 2$ ), and it happened prior to massive fruit drop. On the contrary, auxin responsive, and transport related genes, such as $A u x / I A A$ and $A U X$, were all repressed by GPD. These results agreed with a previous report, where the GPD treatment consistently reduced endogenous auxin content and altered auxin responsive genes in litchi (Kuang et al., 2012). It indicated that auxin signaling and influx were impaired prior to the onset of fruit drop. These results were consistent with other studies on abscission, like mature fruit abscission in melon (Corbacho et al., 2013) and olive

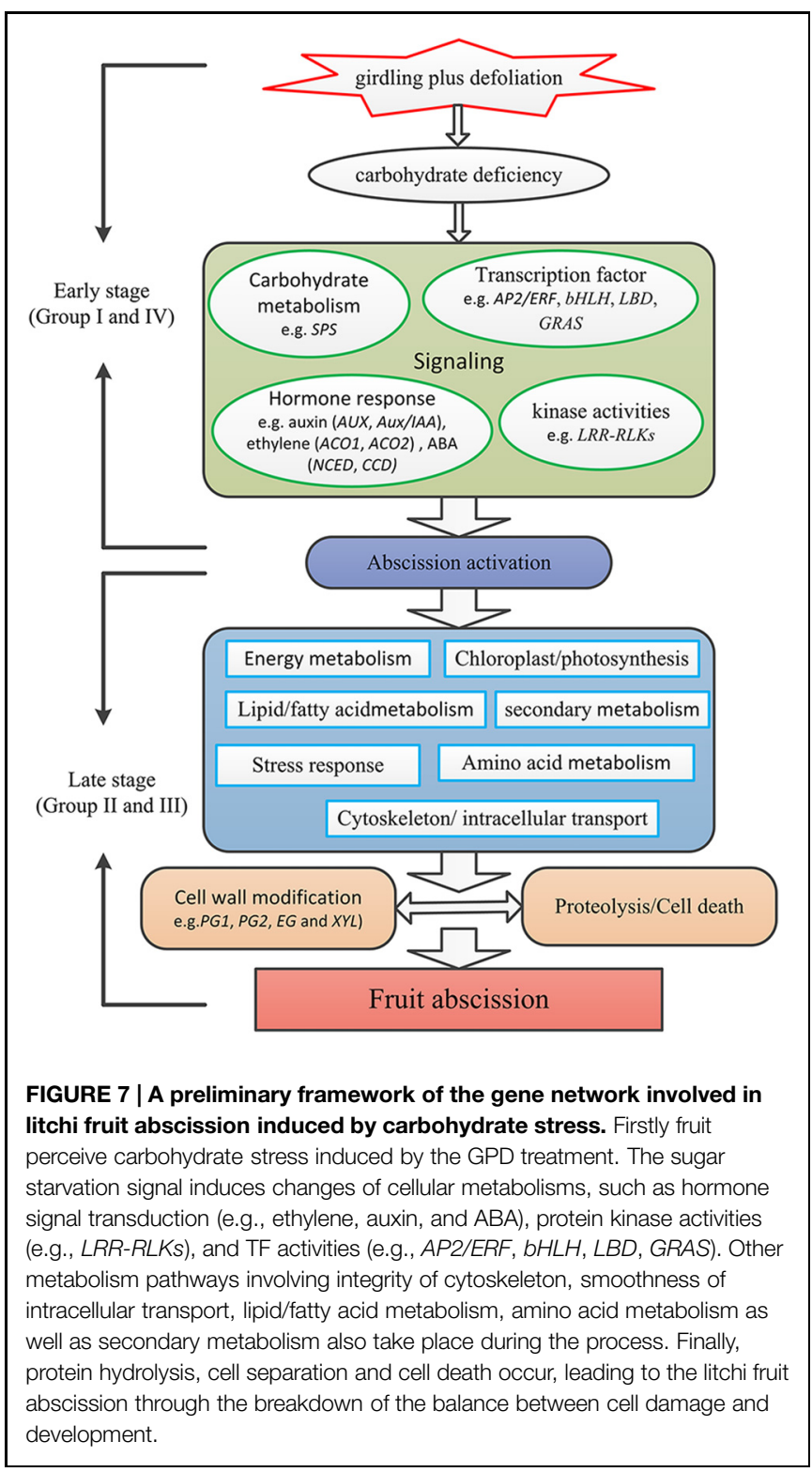


(Gil-Amado and Gomez-Jimenez, 2013), young fruit drop induced by NAA and shading in apple (Zhu et al., 2011). It has been proposed that $\mathrm{ABA}$ might be implicated in response to nutrient stress (Yang et al., 2003), and correlate with the activation of ethylene-associated abscission in citrus fruitlet (Gómez-Cadenas et al., 2000), shading-induced abscission of apple fruitlet (Zhu et al., 2011), and mature abscission in melon fruit (Corbacho et al., 2013). However, we observed a low expression of genes involved in ABA biosynthesis and signaling during the early and/or late stages of fruit abscission in response to the GPD treatment, suggesting that ABA might be involved in litchi fruit abscission through another pathway, rather than integrating with ethylene pathway, as shown in apple (Zhu et al., 2011). In addition, our study indicated that besides the participation of ethylene and auxin in controlling abscission events, other hormones, such as ABA, BR, JA, GA, and cytokinin apparently participated in an intricate interaction network regulating the abscission of GPD-treated fruits.

In Arabidopsis floral abscission (Burr et al., 2011) and melon fruit abscission (Corbacho et al., 2013), LRR receptorlike protein kinase (LRR-RLKs) has been thought to be a potential controller by competing activities. Here, expression analysis detected many LRR-RLKs down-regulated during the whole fruit abscission process induced by the GPD treatment, suggesting the involvement of LRR-RLKs in regulating litchi fruit abscission. In addition, a subset of genes involved in microtubule depolymerization and a group of gene encoding $\mathrm{ABC}$ transporter showed low abundance at 4-7 days after the GPD treatment. These results indicated that changes in integrity of cytoskeleton and the intracellular transport system might be involved in the late stage of litchi fruit abscission. On the other hand, late events were potentially controlled by down-regulation of $A P 2 / E R F$ and GRAS TFs, while the early events may be controlled by downregulation of $A P 2 / E R F, b H L H$, and $L O B$ domain proteins.

The last key step in promoting $A Z$ cell separation that eventually result in the shedding of organs is the induction of cell wall degrading and modifying enzymes, such as PGs, cellulases, xyloglucan endotransglucosylase/hydrolases (XTHs), and expansins (Singh et al., 2011; Zhu et al., 2011; Corbacho et al., 2013). However, not only numerous transcripts related to cell wall biosynthesis but also cell wall degradation and loosening related genes were found to be down-regulated during GPD-induced abscission process. Several cell wall degradation genes, such as $P G 1, E G$, and $X Y L$, was strongly up-regulated, indicating that these genes might play an essential and positive regulation role in cell separation at the early stage of litchi fruit abscission.

Based on our results, a preliminary framework of the gene network involved in litchi fruit abscission was proposed (Figure 7). Fruit may first perceive carbohydrate stress induced by the GPD treatment. Then, the sugar starvation signal transduction might induce changes of cellular metabolisms,

\section{References}

Agustí, J., Merelo, P., Cercós, M., Tadeo, F. R., and Talón, M. (2009). Comparative transcriptional survey between laser-microdissected cells from such as hormone signal transduction (e.g., ethylene, auxin, and $\mathrm{ABA}$ ), protein kinase activities (e.g., $L R R-R L K s$ ), and TF activities (e.g., AP2/ERF, bHLH, LBD, GRAS). Other metabolism pathways could also take place in this process, such as integrity of cytoskeleton, smoothness of intracellular transport, lipid/fatty acid metabolism, amino acid metabolism as well as secondary metabolism. Finally, protein hydrolysis, cell separation, and cell death occur, leading to the litchi fruit abscission through the break of the balance between cell damage and development.

\section{Conclusion}

A litchi fruit transcriptome assembly was greatly improved in this work using Trinity software. 857 GPD-responsive DEGs were identified as candidate genes involved in the process of litchi fruit abscission induced by carbohydrate stress. A hypothetical molecular model for litchi fruit abscission induced by the GPD treatment was proposed based on the results. Our study provided the first information about gene expression profile related to fruit abscission induced by carbohydrate stress on whole transcriptome level, which contributes to a better understanding for the molecular regulatory mechanism of fruit abscission in litchi. Further studies should focus on the functional characterization of genes involved in the above pathways.

\section{Author Contributions}

JL designed the research and finalized the manuscript. CL conceived and drafted this paper. YW performed most of the biological experiments. YW and JL conducted bioinformatics analyses and data interpretation. $\mathrm{XH}$ and $\mathrm{HW}$ revised the manuscript. All the authors approved the final manuscript.

\section{Acknowledgments}

This work was supported by the National Natural Science Foundation of China (31171920) and China Agricultural Research System (CARS-33-11). We are grateful to anonymous reviewers for their comments and Dr. Rui Xia from the Delaware Biotechnology Institute and Department of Plant and Soil Sciences, University of Delaware, USA for his helpful suggestions and critical English language editing.

\section{Supplementary Material}

The Supplementary Material for this article can be found online at: http://journal.frontiersin.org/article/10.3389/fpls. 2015.00439/abstract

laminar abscission zone and petiolar cortical tissue during ethylene-promoted abscission in citrus leaves. BMC Plant Biol. 9:127. doi: 10.1186/1471-2229-9-127 Audic, S., and Claverie, J. M. (1997). The significance of digital gene expression profiles. Genome Res. 7, 986-995. doi: 10.1101/gr.7.10.986 
Berüter, J., and Droz, P. (1991). Studies on locating the signal for fruit abscission in the apple tree. Sci. Hortic. 46, 201-214. doi: 10.1016/0304-4238(91) 90043-X

Botton, A., Eccher, G., Forcato, C., Ferrarini, A., Begheldo, M., Zermiani, M., et al. (2011). Signaling pathways mediating the induction of apple fruitlet abscission. Plant Physiol. 155, 185-208. doi: 10.1104/pp.110.165779

Burr, C. A., Leslie, M. E., Orlowski, S. K., Chen, I., Wright, C. E., Daniels, M. J., et al. (2011). CAST AWAY, a membrane-associated receptor-like kinase, inhibits organ abscission in Arabidopsis. Plant Physiol. 156, 1837-1850. doi: $10.1104 /$ pp.111.175224

Chang, J. C., and Lin, T. S. (2007). Gas exchange in litchi under controlled and field conditions. Sci. Hortic. 114, 268-274. doi: 10.1016/j.scienta.2007.06.023

Chang, J. C., and Lin, T. S. (2008). Fruit yield and quality as related to flushes of bearing shoots in litchi. J. Am. Soc. Hortic. Sci. 133, 284-289.

Cho, S. K., Larue, C. T., Chevalier, D., Wang, H., Jinn, T. L., Zhang, S., et al. (2008). Regulation of floral organ abscission in Arabidopsis thaliana. Proc. Natl. Acad. Sci. U.S.A. 105, 15629-19634. doi: 10.1073/pnas.0805539105

Conesa, A., Gotz, S., Garcia-Gomez, J. M., Terol, J., Talon, M., and Robles, M. (2005). Blast2GO: a universal tool for annotation, visualization and analysis in functional genomics research. Bioinformatics 21, 3674-3676. doi: 10.1093/bioinformatics/bti610

Corbacho, J., Romojaro, F., Pech, J. C., Latche, A., and Gomez-Jimenez, M. C. (2013). Transcriptomic events involved in melon mature-fruit abscission comprise the sequential induction of cell-wall degrading genes coupled to a stimulation of endo and exocytosis. PLoS ONE 8:e58363. doi: 10.1371/journal.pone.0058363

Estornell, L. H., Agusti, J., Merelo, P., Talon, M., and Tadeo, F. R. (2013). Elucidating mechanisms underlying organ abscission. Plant Sci. 199, 48-60. doi: 10.1016/j.plantsci.2012.10.008

Garg, R., Patel, R. K., Tyagi, A. K., and Jain, M. (2011). De novo assembly of chickpea transcriptome using short reads for gene discovery and marker identification. DNA Res. 18, 53-63. doi: 10.1093/dnares/dsq028

Ghangal, R., Chaudhary, S., Jain, M., Purty, R. S., and Chand, S. P. (2013). Optimization of de novo short read assembly of seabuckthorn (Hippophae rhamnoides L.) transcriptome. PLoS ONE 8:e72516. doi: 10.1371/journal.pone. 0072516

Gil-Amado, J. A., and Gomez-Jimenez, M. C. (2013). Transcriptome analysis of mature fruit abscission control in olive. Plant Cell Physiol. 54, 244-269. doi $10.1093 / \mathrm{pcp} / \mathrm{pcs} 179$

Gómez-Cadenas, A., Mehouachi, J., Tadeo, F. R., Primo-Millo, E., and Talon, M. (2000). Hormonal regulation of fruitlet abscission induced by carbohydrate shortage in citrus. Planta 210, 636-643. doi: 10.1007/s0042500 50054

Grabherr, M. G., Haas, B. J., Yassour, M., Levin, J. Z., Thompson, D. A., Amit, I., et al. (2011). Full-length transcriptome assembly from RNA-Seq data without a reference genome. Nat. Biotechnol. 29, 644-652. doi: 10.1038/nbt.1883

Hieke, S., Menzel, C. M., Doogan, V. J., and Ludders, P. (2002). The relationship between yield and assimilate supply in lychee (Litchi chinensis Sonn.). J. Hortic. Sci. Biotechnol. 77, 326-332.

Hu, Z. Q., Wang, H. C., and Hu, G. B. (2005). Measurement of sugars, organic acids and vitamin $\mathrm{C}$ in litchi fruit by high performance liquid chromatography. J. Fruit Sci. 5, 34. doi: 10.3969/j.issn.1009-9980.2005.05.035

Iglesias, D. J., Tadeo, F. R., Primo-Millo, E., and Talon, M. (2006). Carbohydrate and ethylene levels related to fruitlet drop through abscission zone $\mathrm{A}$ in citrus. Trees 20, 348-355. doi: 10.1007/s00468-005-0047-x

Kuang, J. F., Wu, J. Y., Zhong, H. Y., Li, C. Q., Chen, J. Y., Lu, W. J., et al. (2012). Carbohydrate stress affecting fruitlet abscission and expression of genes related to auxin signal transduction pathway in litchi. Int. J. Mol. Sci. 13, 16084-16103. doi: 10.3390/ijms131216084

Li, C. Q., Wang, Y., Huang, X. M., Li, J., Wang, H. C., and Li, J. G. (2013). De novo assembly and characterization of fruit transcriptome in Litchi chinensis Sonn. and analysis of differentially regulated genes in fruit in response to shading. BMC Genomics 14:552. doi: 10.1186/1471-2164-14-552

Li, J. G., Liu, S. Z., and Wang, Z. H. (2004). Changes in endogenous polyamine contents during fruit development of litchi (Litchi chinensis). Plant Physiol. Commun. 40, 153-156.

Li, R., Yu, C., Li, Y., Lam, T. W., Yiu, S. M., Kristiansen, K., et al. (2009). SOAP2: an improved ultrafast tool for short read alignment. Bioinformatics 25, 1966-1967. doi: 10.1093/bioinformatics/btp336
Mehouachi, J., Serna, D., Zaragoza, S., Agusti, M., Talon, M., and Primo-Millo, E. (1995). Defoliation increases fruit abscission and reduces carbohydrate levels in developing fruits and woody tissues of Citrus unshiu. Plant Sci. 107, 189-197. doi: 10.1016/0168-9452(95)04111-7

Meir, S., Philosoph-Hadas, S., Sundaresan, S., Selvaraj, K. S., Burd, S., Ophir, R., et al. (2010). Microarray analysis of the abscission-related transcriptome in the tomato flowerabscission zone in response to auxin depletion. Plant Physiol. 154, 1929-1956. doi: 10.1104/pp.110.160697

Mitra, S. K., Pereira, L. S., Pathak, P. K., and Majumdar, D. (2003). Fruit abscission pattern of lychee cultivars. Acta Hortic. 665, 215-218.

Mortazavi, A., Williams, B. A., McCue, K., Schaeffer, L., and Wold, B. (2008). Mapping and quantifying mammalian transcriptomes by RNA-Seq. Nat. Methods 5, 621-628. doi: 10.1038/nmeth.1226

Moss, G. I., Steer, B. T., and Kriedemann, P. E. (1972). The regulatory role of inflorescence leaves in fruit-setting by sweet orange (Citrus sinensis). Physiol. Plant. 27, 432-438. doi: 10.1111/j.1399-3054.1972.tb03639.x

Peng, G., Wu, J. Y., Lu, W. J., and Li, J. G. (2013). A polygalacturonase gene clustered into clade E involved in lychee fruitlet abscission. Sci. Hortic. 150, 244-250. doi: 10.1016/j.scienta.2012.10.029

Powell, A. A., and Krezdorn, A. H. (1977). Influence of fruit setting treatment on translocation of 14C-metabolites in citrus during flowering and fruiting. J. Am. Soc. Hortic. Sci. 102, 709-714.

Ruiz, R., Garca-Luis, A., Monerri, C., and Guardiola, J. L. (2001). Carbohydrate availability in relation to fruitlet abscission in citrus. Ann. Bot. 87, 805-812. doi: 10.1006/anbo.2001.1415

Schulz, M. H., Zerbino, D. R., Vingron, M., and Birney, E. (2012). Oases: robust de novo RNA-seq assembly across the dynamic range of expression levels. Bioinformatics 28, 1086-1092. doi: 10.1093/bioinformatics/ bts094

Singh, A. P., Tripathi, S. K., Nath, P., and Sane, A. P. (2011). Petal abscission in rose is associated with the differential expression of two ethylene-responsive xyloglucan endotransglucosylase/hydrolase genes, RbXTH1 and RbXTH2. J. Exp. Bot. 62, 5091-5103. doi: 10.1093/jxb/err209

Stern, R. A., Adato, I., Goren, M., Eisenstein, D., and Gazit, S. (1993). Effects of autumnal water stress on litchi flowering and yield in Israel. Sci. Hortic. 54, 295-302. doi: 10.1016/0304-4238(93)90108-3

Stern, R. A., Kigel, J., Tomer, E., and Gazit, S. (1995). 'Mauritius' lychee fruit development and reduced abscission after treatment with the auxin 2,4,5-TP. J. Am. Soc. Hortic. Sci. 120, 65-70.

Talon, M., Tadeo, F. R., Ben-Cheikh, W., Gomez-Cadenas, A., Mehouachi, J., PérezBotella, J., et al. (1997). Hormonal regulation of fruit set and abscission in citrus: classical concepts and new evidence. Acta Hortic. 463, 209-218.

Trueman, S. J., and Turnbull, C. G. N. (1994). Fruit set, abscission and dry matter accumulation on girdled branches of macadamia. Ann. Bot. 74, 667-674. doi: 10.1006/anbo. 1994.1169

Wang, X., Liu, D. M., Li, A. L., Sun, X. L., Zhang, R. Z., Wu, L., et al. (2013). Transcriptome analysis of tomato flower pedicel tissues reveals abscission zonespecific modulation of key meristem activity genes. PLoS ONE 8:e55238. doi: 10.1371/journal.pone.0055238

Wu, J. Y., Li, C. Q., Lu, W. J., and Li, J. G. (2013). Cloning of Lc-ACO1 and its expression related to fruitlet abscission in litchi. J. Fruit Sci. 30, 207-213.

Xiang, X., Qiu, Y. P., and Zhang, Z. W. (1995). Endogenous hormones in the fruit of Litchi chinensis cv. Nuomici relating to fruit abscission. J. Fruit Sci. 12, $88-92$.

Xie, Y. L., Wu, G. X., Tang, J. B., Luo, R. B., Patterson, J., Liu, S. L., et al. (2014). SOAPdenovo-Trans: de novo transcriptome assembly with short RNA-Seq reads. Bioinformatics 30, 1660-1666. doi: 10.1093/bioinformatics/ btu077

Yan, S. C., Chen, J. Y., Yu, W. M., Kuang, J. F., Chen, W. X., Li, X. P., et al. (2011). Expression of genes associated with ethylene-signalling pathway in harvested banana fruit in response to temperature and 1-MCP treatment. J. Sci. Food Agric. 91, 650-657. doi: 10.1002/jsfa.4226

Yang, J. C., Zhang, J. H., Wang, Z. Q., Zhu, Q. S., and Liu, L. J. (2003). Involvement of abscisic acid and cytokinins in the senescence and remobilization of carbon reserves in wheat subjected to water stress during grain filling. Plant Cell Environ. 26, 1621-1631. doi: 10.1046/j.1365-3040.2003.01081.x

Yuan, R. C., and Huang, H. B. (1988). Litchi fruit abscission: its patterns, effect of shading and relation to endogenous abscisic acid. Sci. Hortic. 36, 281-292. doi: 10.1016/0304-4238(88)90063-5 
Yuan, R. C., and Huang, H. B. (1992). Improvement of fruit-set in Litchi chinensis Sonn. through regulation of source-sink relationships. J. S. China Agric. Univ. 13, 136-141.

Yuan, R. C., and Huang, H. B. (1993). Regulation of root and shoot growth and fruit drop of young litchi trees by trunk girdling in view of source-sink relationship. J. Fruit Sci. 10, 195-198.

Yuan, W. Q., Huang, X. M., Wang, H. C., Li, J. G., Chen, H. B., and Yin, J. H. (2009). Seasonal changes in carbon nutrition reserve in Nuomici Litchi trees and its relation to fruit load. Acta Hortic. Sinica 35, 1533-1538.

Zerbino, D. R., and Birney, E. (2008). Velvet: algorithms for de novo short read assembly using de Bruijn graphs. Genome Res. 18, 821-829. doi: 10.1101/gr.074492.107

Zhang, J. Z., Zhao, K., Ai, X. Y., and Hu, C. G. (2014). Involvements of PCD and changes in gene expression profile during self-pruning of spring shoots in sweet orange (Citrus sinensis). BMC Genomics 15:892. doi: 10.1186/1471-216415-892

Zhao, Q. Y., Wang, Y., Kong, Y. M., Luo, D., Li, X., and Hao, P. (2011). Optimizing de novo transcriptome assembly from short-read RNA-Seq data: a comparative study. BMC Bioinformatics 12(Suppl. 14):S2. doi: 10.1186/1471-2105-12S14-S2
Zhou, X. J., Yan, H. D., Bai, H. H., and Yao, W. D. (1999). Carbohydrate and endohormone status in relation to fruit set as influenced by trunk spiral girdling of young litchi trees. Acta Hortic. Sinica 26, 77-80. doi: 10.3321/j.issn:0513353X.1999.02.002

Zhu, H., Dardick, C. D., Beers, E. P., Callanhan, A. M., Xia, R., and Yuan, R. C. (2011). Transcriptomics of shading-induced and NAA-induced abscission in apple (Malus domestica) reveals a shared pathway involving reduced photosynthesis, alterations in carbohydrate transport and signaling and hormone crosstalk. BMC Plant Biol. 11:138. doi: 10.1186/1471-2229-11-138

Conflict of Interest Statement: The authors declare that the research was conducted in the absence of any commercial or financial relationships that could be construed as a potential conflict of interest.

Copyright (c) $2015 \mathrm{Li}$, Wang, Huang, Li, Wang and Li. This is an open-access article distributed under the terms of the Creative Commons Attribution License (CC BY). The use, distribution or reproduction in other forums is permitted, provided the original author(s) or licensor are credited and that the original publication in this journal is cited, in accordance with accepted academic practice. No use, distribution or reproduction is permitted which does not comply with these terms. 